01

\title{
Метод релятивистской синхронизации мобильных атомных часов \\ и его экспериментальная проверка
}

\author{
() В.Ф. Фатеев, Е.А. Рыбаков, Ф.Р. Смирнов
}

Всероссийский научно-исследовательский институт физико-технических и радиотехнических измерений, Московская область, Менделеево

E-mail: fateev@vniiftri.ru, rybakov@vniiftri.ru

Поступило в Редакцию 27 июля 2016 г.

Приведены результаты эксперимента по компенсации релятивистских эффектов смещения шкалы времени транспортируемых водородных часов с нестабильностью $3 \cdot 10^{-15}$ на трассе их движения. Расчет релятивистского ухода бортовой шкалы транспортируемых часов производился на основе непрерывного измерения их текущих координат и скорости с помощью навигационной аппаратуры потребителя ГЛОНАCC/GPS вдоль трассы движения. Методическая погрешность эксперимента не превышает \pm 1 ps.

DOI: 10.21883/PJTF.2017.10.44614.16624

C повышением стабильности атомных мобильных часов до $10^{-15}-10^{-17}$ встает проблема высокоточной компенсации релятивистских уходов их шкал времени на трассе движения [1-4]. Метод релятивистской синхронизации на основе непрерывного вычисления этих эффектов вдоль трассы движения путем измерения их координат и скорости с помощью систем ГЛОНАCС/GPS предложен в работе [5].

Релятивистский эффект смещения времени $\Delta \tau_{G R}$ изменяющийся вдоль трассы перемещения часов, определяется из следующего соот- 
ношения [5]:

$$
\Delta \tau_{G R}=\Delta \tau_{G R}^{0}+\Delta \tau_{G R}^{M}+\Delta \tau_{\Omega}^{M}+\Delta \tau_{V}^{M}+\Delta \tau_{S}^{M},
$$

где

$$
\begin{aligned}
\Delta \tau_{G R}^{0} & =\frac{1}{c^{2}} \int_{\tau_{01}}^{\tau_{02}}\left[\left(\varphi_{0}^{n}+\varphi_{0}^{a n}\right)+\frac{1}{2} \Omega^{2}\left(x_{0}^{2}+y_{0}^{2}\right)\right] d \tau_{0} \\
& =\frac{\Delta \tau_{0}}{c^{2}}\left[\left(\varphi_{0}^{n}+\varphi_{0}^{a n}\right)+\frac{1}{2} \Omega^{2}\left(x_{0}^{2}+y_{0}^{2}\right)\right]
\end{aligned}
$$

- линейно нарастающая составляющая гравитационного смещения, определяемая положением базовых часов; $c-$ скорость света; $\varphi_{0}^{n}$, $\varphi_{0}^{a n}-$ нормальная и аномальная составляющая потенциала стационарных часов; $\Omega=7.29 \cdot 10^{-5} \mathrm{~s}^{-1}-$ угловая скорость вращения Земли; $x_{0}$ и $y_{0}-$ координаты стационарных часов; $\tau_{0}-$ текущее время по шкале стационарных часов, отсчитываемое от момента начала эксперимента $\tau_{01}$ до его окончания $\tau_{02}$;

$$
\Delta \tau_{G R}^{M}=\frac{1}{c^{2}} \int_{\tau_{01}}^{\tau_{02}}\left(\varphi_{m}^{n}\left(\tau_{0}\right)+\varphi_{m}^{a n}\left(\tau_{0}\right)\right) d \tau_{0}
$$

- гравитационное смещение шкалы времени, определяемое текущим гравитационным потенциалом, изменяющимся вдоль трассы перемещения мобильных часов;

$$
\varphi_{m}^{n}=(\mu / \rho)\left[1-J_{2}\left(R_{e} / \rho\right) P_{2}(\sin \psi)\right]
$$

- нормальная составляющая потенциала, определяемая нулевой и второй зональной гармоникой разложения потенциала с коэффициентом $J_{2}=1.0826 \cdot 10^{-3} ; P_{2}(\sin \psi)=\frac{3}{2} \sin ^{2} \psi-\frac{1}{2}-$ полином Лежандра; $\psi$ - геоцентрическая широта; $\mu=3.986 \cdot 10^{14} \mathrm{~m}^{3} / \mathrm{s}^{2}-$ геоцентрическая гравитационная постоянная; $\rho\left(\tau_{0}\right)=\sqrt{x\left(\tau_{0}\right)^{2}+y\left(\tau_{0}\right)^{2}+z\left(z_{0}\right)^{2}}-$ геоцентрическое расстояние точек трассы часов; $R_{e}=6.378 \cdot 10^{6} \mathrm{~m}-$

Письма в ЖТФ, 2017, том 43, вып. 10 
экваториальный радиус Земли (большая полуось референц-эллипсоида);

$$
\begin{aligned}
\varphi_{m}^{a n} & =\frac{\mu}{\rho\left(\tau_{0}\right)}\left[-\sum_{n=3}^{\infty} J_{n}\left(\frac{R_{e}}{\rho\left(\tau_{0}\right)}\right)^{n} P_{n}(\sin \psi)\right. \\
& \left.+\sum_{n=2}^{\infty} \sum_{m=1}^{n}\left(\frac{R_{e}}{\rho\left(\tau_{0}\right)}\right)^{n}\left(C_{n m} \cos (m \lambda)+S_{n m} \sin (m \lambda)\right) P_{n m}(\sin \psi)\right]
\end{aligned}
$$

- аномальная составляющая потенциала мобильных часов, включающая зональные гармоники $J_{n}$, начиная с третьей, а также секториальные и тессеральные гармоники разложения потенциала по сферическим функциям с коэффициентами $C_{n m}, S_{n m} ; P_{n m}(\sin \psi)$ - присоединенные полиномы Лежандра; $\lambda$ - долгота рассматриваемой точки;

$$
\Delta \tau_{\Omega}^{M}=-\frac{1}{c^{2}} \int_{\tau_{01}}^{\tau_{02}}\left[\frac{\Omega^{2}}{2}\left(x_{m}^{2}\left(\tau_{0}\right)+y_{m}^{2}\left(\tau_{0}\right)\right)\right] d \tau_{0}
$$

- релятивистское смещение, определяемое центробежным гравитационным потенциалом мобильных часов;

$$
\Delta \tau_{V}^{M}=-\frac{1}{c^{2}} \int_{\tau_{01}}^{\tau_{02}} \frac{1}{2} V\left(\tau_{0}\right)^{2} d \tau_{0}
$$

- релятивистское смещение, определяемое эффектом Доплера второго порядка; $V\left(\tau_{0}\right)$ - скорость атомных часов относительно поверхности Земли;

$$
\Delta \tau_{S}^{M}=-\frac{\Omega}{c^{2}} \int_{\tau_{01}}^{\tau_{02}}\left(x_{m}\left(\tau_{0}\right) V_{y}\left(\tau_{0}\right)-y_{m}\left(\tau_{0}\right) V_{x}\left(\tau_{0}\right)\right) d \tau_{0}
$$

- релятивистское смещение, определяемое эффектом Саньяка.

Задача определения релятивистских эффектов смещения времени в мобильных атомных часах заключается в вычислении всех составляющих выражения (1) методом численного интегрирования по измерениям текущих координат и скорости с помощью НАП ГЛОНАCC/GPS.

Оценим погрешности всех составляющих, входящих в выражение (1).

Письма в ЖТФ, 2017, том 43, вып. 10 
Поскольку $J_{2}=1.0826 \cdot 10^{-3}$, то погрешность определения второго слагаемого в выражении нормального потенциала в $\Delta \tau_{G R}^{M}$ (3) пренебрежимо мала. Тогда нормальный потенциал можно представить в виде $\varphi_{m}^{n}=\mu / \rho$ и максимальная погрешность его вычисления будет иметь следующий вид:

$$
\begin{aligned}
\delta\left(\Delta \tau_{\varphi}^{M}\right) & \approx-\frac{\mu}{c^{2} R_{e}^{3}} \sum_{i=0}^{n-1}\left(\delta_{x} \frac{x_{i+1}+x_{i}}{2}\left(t_{i+1}-t_{i}\right)\right. \\
& \left.+\delta_{y} \frac{y_{i+1}+y_{i}}{2}\left(t_{i+1}-t_{i}\right)+\delta_{z} \frac{z_{i+1}+z_{i}}{2}\left(t_{i+1}-t_{i}\right)\right),
\end{aligned}
$$

где $\delta_{x}, \delta_{y}, \delta_{z}$ - ошибки измерения координат $x_{i}, y_{i}$ и $z_{i}$ соответственно. Будем считать ошибки измерения координат с помощью навигационной аппаратуры потребителя (НАП) постоянными вдоль трассы.

Погрешности определения координат навигационных приемников, использующих режим Real Time Kinematic (RTK), составляет $15 \mathrm{~cm}$ [6]. Тогда при движении мобильных атомных часов в течение $12 \mathrm{~h}$ максимальная погрешность составляет около 1 ps.

Для компенсации релятивистских эффектов аномальной составляющей потенциала при вычислении смещения $\Delta \tau_{G R}^{M}$ с точностью не хуже $1 \mathrm{ps}$ необходимо учитывать гармоники $C_{n m}$ и $S_{n m}$, значения которых не превышают $33 \cdot 10^{-9}$. Для модели поля EIGEN-6C4 [7] это соответствует гармоникам порядка и степени $20 \times 20$.

Абсолютная погрешность величины релятивистского смещения времени $\Delta \tau_{G R}^{M}$, вызванного влиянием центробежного потенциала, определяется выражением, следующим из (3):

$$
\delta\left(\Delta \tau_{\Omega}^{M}\right) \approx \frac{\Omega^{2}}{c^{2}} \sum_{i=0}^{n-1}\left[\delta x \frac{x_{i+1}+x_{i}}{2}\left(t_{i+1}-t_{i}\right)+\delta y \frac{y_{i+1}+y_{i}}{2}\left(t_{i+1}-t_{i}\right)\right] .
$$

При продолжительности интервала движения мобильных атомных часов не более $12 \mathrm{~h}$ и с учетом погрешностей определения координат, указанных выше, погрешность не будет превышать значения $10^{-2} \mathrm{ps}$.

Абсолютная погрешность определения релятивистского расхождения шкал, вызванного влиянием эффекта Доплера второго порядка,

Письма в ЖТФ, 2017, том 43, вып. 10 
определяется выражением, следующим из (7):

$$
\begin{aligned}
\delta\left(\Delta \tau_{V}\right) & =\frac{1}{c^{2}} \int_{\tau_{01}}^{\tau_{02}} V \delta V d \tau_{0} \\
& \approx \frac{1}{c^{2}} \sum_{i=1}^{n-1}\left[\delta V \frac{V_{i+1}+V_{i}}{2}\left(t_{i+1}-t_{i}\right)\right],
\end{aligned}
$$

где $V_{m}=\left\{V_{1}, V_{2}, \ldots, V_{n}\right\}-$ массив измерений скорости подвижных атомных часов, значения которой определены в моменты $t_{m}=\left\{t_{1}, t_{2}, \ldots, t_{n}\right\}$ (измерения по НАП).

Погрешность определения скорости навигационным приемником TRIMBLE BX982 составляет $2 \cdot 10^{-2} \mathrm{~m} / \mathrm{s}$ [6]. Тогда при скорости атомных часов не более $30 \mathrm{~m} / \mathrm{s}(108 \mathrm{~km} / \mathrm{h})$ погрешность синхронизации, вызванная движением хранителя времени, будет не более 0.3 ps при движении в течение $12 \mathrm{~h}$.

Погрешность вычисления релятивистского расхождения шкал, вызванного эффектом Саньяка (8), определяется следующим выражением:

$$
\begin{aligned}
\delta\left(\Delta \tau_{S}^{M}\right) & \approx-\frac{\Omega}{c^{2}} \sum_{n=1}^{n-1}\left[\left(\frac{V_{y_{i+1}}+V_{y_{i}}}{2} \delta x+\frac{x_{i+1}+x_{i}}{2} \delta V_{y}\right.\right. \\
& \left.\left.-\frac{y_{i+1}+y_{i}}{2} \delta V_{x}-\frac{V_{x_{i+1}}+V_{x_{i}}}{2} \delta y\right)\left(t_{i+1}-t_{i}\right)\right] .
\end{aligned}
$$

При наихудших условиях оценки этой ошибки, когда часы движутся поперек меридиана $\left(x=R_{e}, y=0, V_{x}=0, V_{y}=30 \mathrm{~m} / \mathrm{s}\right)$, максимально возможная погрешность синхронизации, вызванная эффектом Саньяка, не превышает 9 ps. Однако, если часы движутся вдоль меридиана $\left(x=R_{e}, y=0, V_{x}=30 \mathrm{~m} / \mathrm{s}, V_{y}=0\right)$, ошибка равна нулю. Другая возможность уменьшения эффекта Саньяка и соответствующей ошибки синхронизации заключается в использовании маршрута часов „туда и обратно“ и прокладке обеих частей маршрута по одному и тому же пути. Это объясняется тем, что данный эффект пропорционален площади проекции треугольника с вершинами в точках $0, \mathbf{R}_{m}, \mathbf{R}_{0}\left(\mathbf{R}_{m}\right.$ и $\mathbf{R}_{0}$ - радиус-вектор положения мобильных и стационарных часов

Письма в ЖТФ, 2017, том 43, вып. 10 


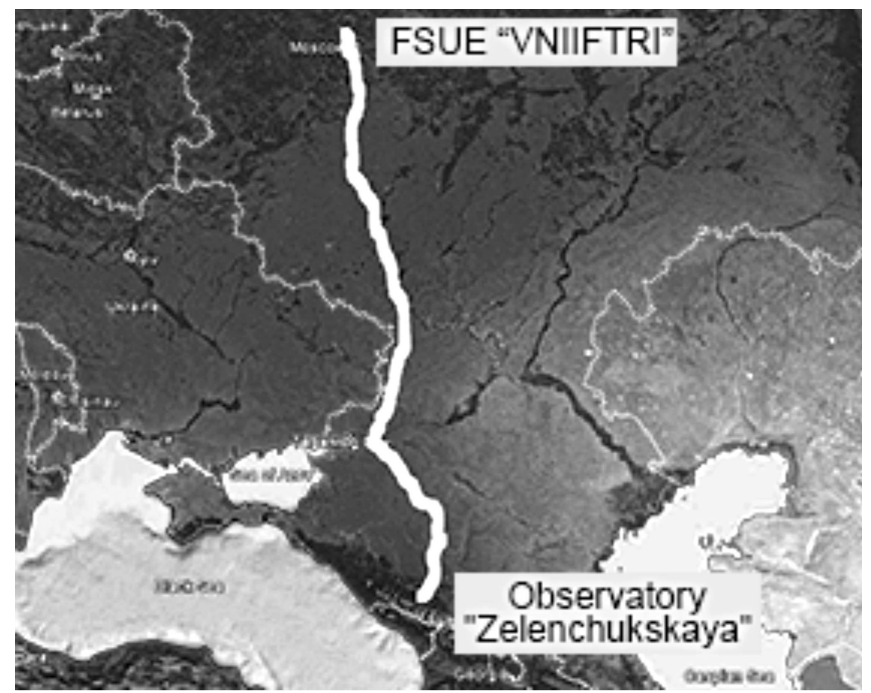

Рис. 1. Маршрут движения водородного стандарта частоты и времени Ч1-76А.

соответственно во вращающейся земной системе отсчета) на плоскость экватора, а его знак зависит от направления движения [5].

Экспериментальная проверка метода релятивистской синхронизации заключалась в вычислении релятивистских эффектов смещения шкалы времени водородного стандарта частоты и времени Ч1-76А на маршруте: ВНИИФТРИ (г.п. Менделеево, Московская обл.) - Радиоастрономическая обсерватория (РАО) „Зеленчукская“ (ст. Зеленчукская, респ. Карачаево-Черкессия) (рис. 1).

Расчет релятивистских эффектов по маршруту движения в интересах их компенсации осуществлялся по формулам (1)-(12) с использованием программного продукта MATLAB на основе измерений текущих координат и скорости, выполненных с помощью приемника Javad Sigma. В расчетах применялась модель разложения гравитационного поля EIGEN-6C4. На рис. 2 и 3 показано, как изменялись вдоль трассы суммарное релятивистское расхождение шкал времени мобильного и стационарного стандарта частоты и времени и текущая ошибка синхронизации соответственно.

Письма в ЖТФ, 2017, том 43, вып. 10 


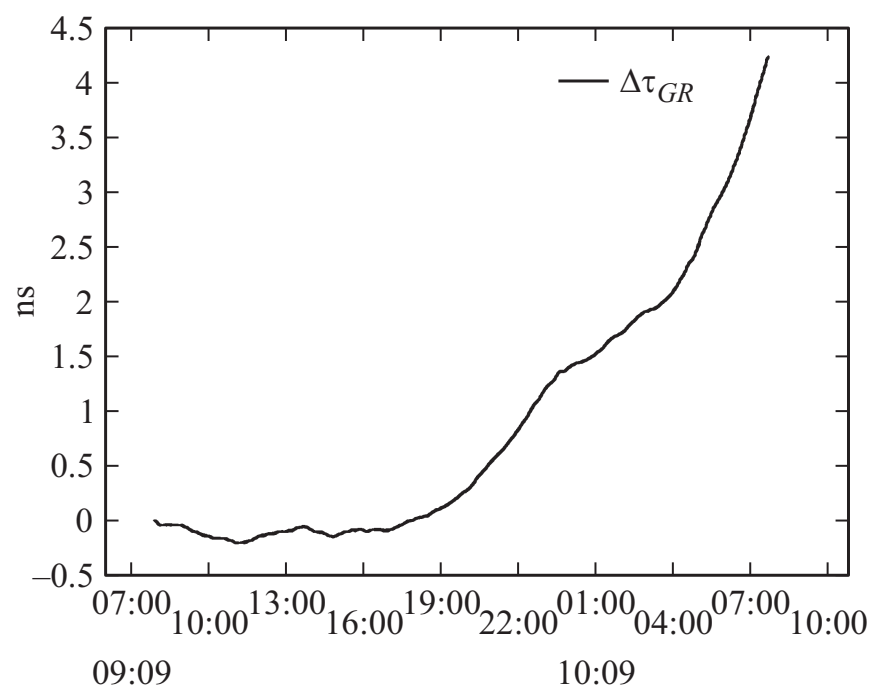

Рис. 2. Текущее релятивистское гравитационное смещение времени вдоль маршрута движения ВНИИФТРИ - РАО „Зеленчукская“.

На момент окончания движения вычисленное суммарное релятивистское расхождение шкалы времени составило: $\Delta \tau_{\text {relGNSS }}=+4.241 \mathrm{ns,}$ ошибка вычисления $\delta \tau_{r e l} \pm 0.001 \mathrm{~ns}$.

Для верификации метода релятивистской синхронизации использовалась синхронизация стандартов частоты и времени (подвижного и стационарного) путем измерения расхождения их шкал на концах маршрута. Расхождение шкал двух стандартов частоты $\Delta \tau$ в конце маршрута движения определяется следующим выражением [8]:

$$
\Delta \tau=\Delta \tau_{r e l}+\Delta \tau_{\Delta f}+\Delta \tau_{\mathrm{TCF}} \pm \sigma_{\Delta r},
$$

где $\Delta \tau_{r e l}-$ расхождение за счет релятивистских эффектов; $\Delta \tau_{\Delta f}-$ расхождение шкал времени за счет начального расхождения частот стандартов $\Delta f / f$, которое измеряется непосредственно перед поездкой; $\Delta \tau_{\mathrm{TCF}}$ - расхождение шкал за счет разности температур в точках размещения стандартов, которое рассчитывается через температурный коэффициент частоты; $\sigma_{\Delta \tau}-$ среднеквадратическая ошибка измерений.

Письма в ЖТФ, 2017, том 43, вып. 10 


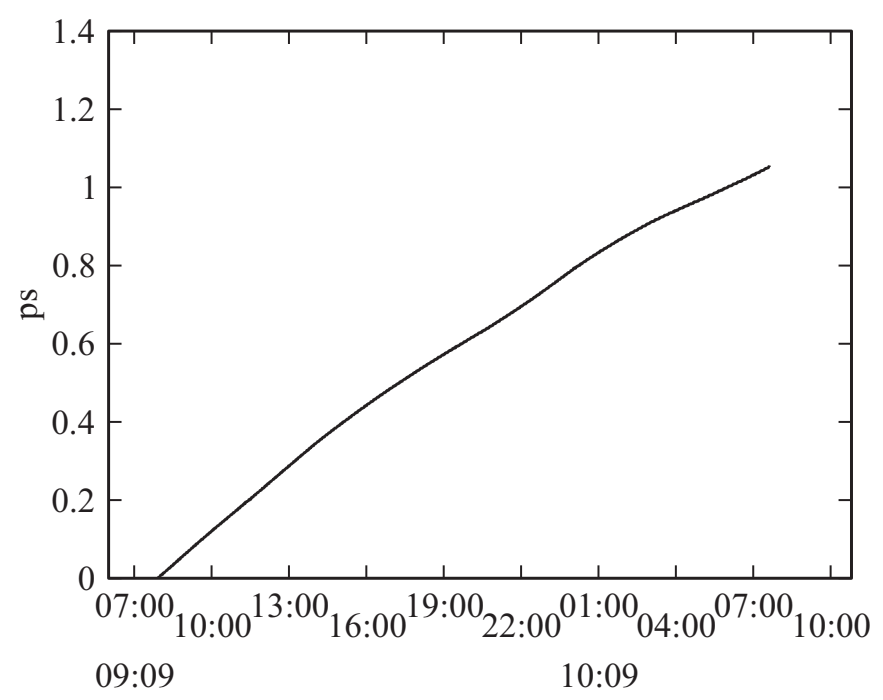

Рис. 3. Суммарная текущая ошибка вычисления релятивистского гравитационного смещения времени вдоль маршрута движения ВНИИФТРИ - РАО „Зеленчукская“.

По результатам измерений с помощью приемников GTR51 определяется $\Delta \tau$ и по расчетным значениям $\Delta \tau_{\Delta f}, \Delta \tau_{\mathrm{TCF}}, \sigma_{\Delta \tau}$ вычисляется искомый гравитационный эффект $\Delta \tau_{r e l}$, который в конце маршрута ВНИИФТРИ - РАО „Зеленчукская“ составляет $\Delta \tau_{r e l}=5.1 \pm 1.5 \mathrm{~ns}$.

Таким образом, вычисленное значение релятивистских эффектов смещения шкал времени по методу релятивистской синхронизации составляет $4.241 \pm 0.001 \mathrm{~ns}$ и находится внутри интервала неопределенности независимого метода синхронизации, имеющего погрешность \pm 1.5 .

Предлагаемый метод на основе использования НАП ГЛОНАCC/GPS целесообразно использовать для синхронизации создаваемых в настоящее время в России и других странах оптических стандартов частоты и времени с нестабильностью $10^{-17}$ и лучше. В представленном методе релятивистской синхронизации подвижные атомные часы соответствующей стабильности синхронизированы с базовыми часами с пикосекундной точностью в любой точке трассы без использования специальных линий связи между ними.

Письма в ЖТФ, 2017, том 43, вып. 10 


\section{Список литературы}

[1] Hafele J., Keating R. // Science. 1972. V. 177. P. 166-168.

[2] Борисова Л.Б., Мельников В.Н. // Измерительная техника. 1988. № 4. С. $13-$ 15. [Borisova L.B., Mel'nikov V.N. // Meas. Techniq. 1988. V. 31. N 4. P. 323-327.]

[3] Медведев Ю.Н., Смирнов Ю.Ф. // Метрология времени пространства: Тр. 5-го Рос. симп. М.: ВНИИФТРИ, 1994. С. 342-343.

[4] Гайгеров Б.А., Сысоев В.П. // Измерительная техника. 2012. № 2. С. 25-29.

[5] Фатеев В.Ф., Сысоев В.П. // Измерительная техника. 2014. № 8. С. 31-35. [Fateev V.F., Sysoev V.P. // Meas. Techniq. 2014. V. 57. N 8.]

[6] TRIMBLE BX982 http:/trl.trimble.com/ docushare/dsweb/Get/Document-581007

[7] Förste C., Bruinsma S., Abrikosov O. et al. EIGEN-6C4. The latest combined global gravity field model including GOCE data up to degree and order 2190 of GFZ. Potsdam and GRGS Toulouse, 2014.

[8] Фатеев В.Ф., Сысоев В.П., Рыбаков Е.А. // Измерительная техника. 2016. № 4. C. 41-43.

Письма в ЖТФ, 2017, том 43, вып. 10 